\title{
Traditional ecological knowledge of the bird traders on bird species bird naming, and bird market chain: A case study in bird market Pasty Yogyakarta, Indonesia
}

\author{
JOHAN ISKANDAR ${ }^{1}$, BUDIAWATI S. ISKANDAR ${ }^{2}$, DEDE MULYANTO ${ }^{2}$, RACHMAN LATIF ALFIAN ${ }^{2}$, \\ RUHYAT PARTASASMITA, ${ }^{1, \varphi}$ \\ ${ }^{1}$ Department of Biology, Faculty of Mathematics and Natural Sciences, Universitas Padjadjaran. Jl. Raya Bandung Sumedang Km. 21, Jatinangor, \\ Sumedang 45363, West Java, Indonesia. Tel.: +62-22-7796412, Fax.: +62-22-7796412, . `email: ruhyat.partasasmita@unpad.ac.id; \\ rp2010rikkyo@gmail.com \\ ${ }^{2}$ Department of Anthropology, Faculty of Social Science and Political Science, Universitas Padjadjaran. Jl. Raya Bandung Sumedang Km. 21, Jatinangor, \\ Sumedang 45363, West Java, Indonesia
}

Manuscript received: 11 April 2020. Revision accepted: 16 May 2020

\begin{abstract}
Iskandar J, Iskandar BS, Mulyanto D, Alfian RL, Partasasmita R. 2020. Traditional ecological knowledge of the bird traders on bird species bird naming, and bird market chain: A case study in bird market Pasty Yogyakarta, Indonesia. Biodiversitas 21: 25862602. The bird-keeping in Indonesian society has been popular for a long time. Because the demand for birds has increased in an urban area, plenty of bird species are predominantly traded in urban bird markets. Bird market is a place for selling and buying various birds. The rise of bird trade in the bird markets in many regions of Indonesia has provided social and economic benefits for many people. But in addition to providing many benefits, the plentiful bird trade in bird markets that are not well managed is very worrying about the extinction of various bird species in rural ecosystems. This paper aimed to elucidate the Traditional Ecological Knowledge (TEK) of bird traders on bird species traded, bird naming and folk classification of bird species, and market chain system based on a case study in bird market of Pasty, Yogyakarta. The method used in this study was a qualitative method with the ethnoornithological approach. The results of the study showed that it was documented 106 bird species representing 40 families traded in the Pasty bird market. The common local names and folk bird classification are culturally based on bird vocalization, special colors, morphological characteristics, special habitats, involvement in bird song contests, flying racing competitions, selling prices, and wilderness. Most wild and semi-bird species are traded in the Pasty bird market are predominantly supplied from a rural ecosystem.
\end{abstract}

Keywords: Bird market, market chain system, traditional ecological knowledge, Yogyakarta

\section{INTRODUCTION}

Based on ecological history, in the past lot of people in Indonesia hobby kept live birds in cages based on Traditional Ecological Knowledge (TEK) and strongly embedded with culture (Iskandar 2015). However, unlike in the past, today birds are kept by many people of both rural and urban areas are mainly not only for the hobby but also for trading commodities (Jepson 2010). In addition, a hobby of bird keeping for some people is mainly used for competing in bird song contests (Iskandar and Iskandar 2015). Since the demand for keeping live birds has increased, the bird markets have been occurring in many regions of Indonesia. Bird market is a place for selling and buying various birds. Therefore, the bird markets function as hallmarks of a particular culture or society by reproducing, on a small scale, the cultural and bird diversity of a region (Alburqueque et al. 2014).

The rise of bird trade in bird markets in many urban regions of Indonesia has provided some social and economic benefits for many people. For example, in addition, to sell various birds, by developing bird trade in the bird market has developed trading various other commodities, including natural bird feed, artificial bird feed, bird vitamins and medicines, bird cages, nest boxes, and various bird nest materials that create jobs and provide income for many people.

In addition to providing many benefits, the plentiful bird trade in bird markets that are not well managed is very worrying about the extinction of bird species in rural ecosystems. This is because most wild or semi-wild birds are commonly traded in the bird market that is mainly obtained from illegal hunting in rural ecosystems. Today, almost each bird species can be sold in the market. An endangered species of bird in the ecosystem, the selling price in the bird market is increasingly expensive. Consequently, villagers are increasingly want to hunt and sell them to get money. The birds' species that are commonly involved in the local completion of the bird song contests have predominantly non-breeding species, indicated did not have a ring in their feet. Therefore, because the bird species has been popularly contested in the songbird competition, the price is higher (Iskandar and Iskandar 2015). Based on empirical data, in the last decades, some birds that are predominantly contested in the bird song competition and intensively hunted by village people have dramatically decreased of songbird populations; the cucak rowo/straw-headed bulbul 
(Pycnonotus zeylanicus) is a case in point (Iskandar and Iskandar 2015). Therefore, in last several decades, it has been revealed that change or loss habitat and capturing wild birds for commercial purposes across culture in the world have been the principal causes of population decrease among many bird species (Bennet 2002; Suba et al. 2011; van Balen et al. 2011; Harris et al. 2015; Chng et al. 2015; Ayat and Tata 2015; Irawati et al. 2015; Iskandar 2014; Iskandar et al. 2016; Partasasmita et al. 2016; Dai and $\mathrm{Hu}$ 2017; Shepherd and Cassey 2017; Setioko 2019; Hakim et al. 2020).

In terms of bird conservation, Indonesia has a law to conserve natural resources, including species of birds, i.e. Law No. 5 of 1990 concerning biological natural resources and their ecosystems. Based on this law, it is explained that the species of birds protected by the law are not allowed to hunt, injure, maintain, transport, and trade in a condition of life and death (Department of Forestry 2007). However, in practice, many species of birds that have been protected by this law are predominantly traded in the bird market. This is partly due to the absence of strict action from the government, as well as a lack of awareness from the public. Other weakness of conservations including miss conception. The nature conservation is linked to the people that are direct to people's values and behavior, therefore, the people-conservation interface must be seriously given attention (Cunningham 2001) Traditional bird markets as an arena where bird traders and buyers meet so that they create a system local culture of human relationships with bird species and their environment, and the diversity of bird species in the region and its surroundings (Albuquerque et al. 2014).

Some studies on bird markets, such as diversity of bird species, bird prices, and bird origins have been carried out by many researchers (Widodo 2007; Bashari and Nurdin 2009; Suba et al. 2011; Juniawati 2012; Chng et al. 2015; Prakosa and Iriawan 2015; Shepherd and Cassey 2017; Iskandar et al. 2019; Setioko 2019). However, studies on birds in the bird markets that is based on ethnoornithological approach are still rare. Ethnoornithology is a branch of ethnozoology that is the scientific study of the relationships between people and birds in culture or the study of local knowledge of birds embedded with culture (Hunn 2011; Alves and Souto 2015; Pam 2017). It has evolved over the past several decades bringing together nature conservation and local people and Traditional Ecological Knowledge (TEK) which is potentially a useful tool for engaging people from various cultures into biodiversity conservation (Pam 2017). Similarly, according to Alves (2012), ethnoornithological and ethnozoological studies can be a valuable asset to increase our standing of the cultural, economic, social, and traditional roles played by animals, including birds. In this context, they have a central role in conservation and management.

This paper aimed to elucidate the Traditional Ecological Knowledge (TEK) of bird traders on bird species traded, bird naming and folk classification of bird species, and market chain system based on case study in Bird Market of Pasty, Yogyakarta.

\section{MATERIALS AND METHODS}

\section{Study sites}

This study was undertaken in bird market of Pasty (Pasty: Pasar Satwa dan Tanaman Hias YogyakartaAnimal and Ornamental market of Yogyakarta) on August 2019 and February 2020. The bird market of Pasty is located in Jalan Bantul Km 1, No. 141, Dongkelan, Subdistrict of Mantri Jeron, Province of Yogyakarta, Indonesia (Figure 1). Geographically Yogyakarta is located approximately between latitudes of $7^{0} 15^{\prime} 24^{\prime \prime}$ and $7^{0} 49^{\text {' }}$ $30^{\prime \prime}$ South and between longitudes of $110^{\circ} 24^{\prime} 19^{\prime \prime}$ and $110^{\circ}$ 28 ' 53" east. It was relocated from the traditional market of Pasar Ngasem that is inaugurated on April 22, 2010.

Bird market of Pasty has total area of $29.635 \mathrm{~m}^{2}$ consists of two zones: East Zone and West Zone. The East Zone is mainly used for placing of the trading animals, while the West Zone is functioned for locating of the trading flora (Figure 2).

The east zone consists of some buildings, including an animal health post $\left(35 \mathrm{~m}^{2}\right), 16$ stalls $\left(2276 \mathrm{~m}^{2}\right), 39$ nonpermanent stalls $\left(39 \mathrm{~m}^{2}\right), 1$ bird vault $\left(65.5 \mathrm{~m}^{2}\right), 5$ toilets $\left(108 \mathrm{~m}^{2}\right), 1$ praying place $\left(55 \mathrm{~m}^{2}\right), 1$ security post $\left(13 \mathrm{~m}^{2}\right)$, and 3 composters. Permanent stalls (kios) and nonpermanent stalls are classified based commodities traded, including birds; bird feed; birdcages; decorative fishes; feed of decorative fish; and other animals, including cat, dog, rabbit, hamster, monkey, turtle, and reptiles.

Bird traders are documented 214 people consisting of dominant bird traders and other wildlife traders (Adinugroho 2013). The species of birds traded in the Pasty bird market are usually kept in cages placed inside stalls. Every day the birds' cages are taken out from installs and hung in front of stall, so birds may get the morning sun. But during the day the bird cages are moved to places where there is no sunlight. Meanwhile, in the afternoon the birdcages were put into the stall, and the stall is closed.

In addition to bird species, various utensils, including cages, nest boxes, wooden stick bird perches, bamboo bird perches, bird foods, and bird vitamin and medicine are sold in stalls (Figure 3).

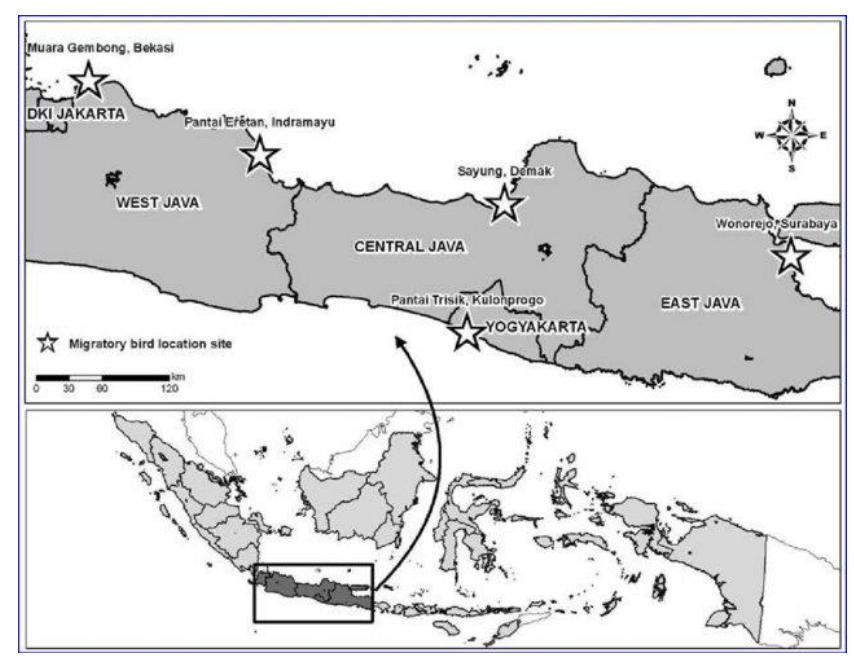

Figure 1. Study site at bird market of Pasty Yogyakarta, Indonesia 


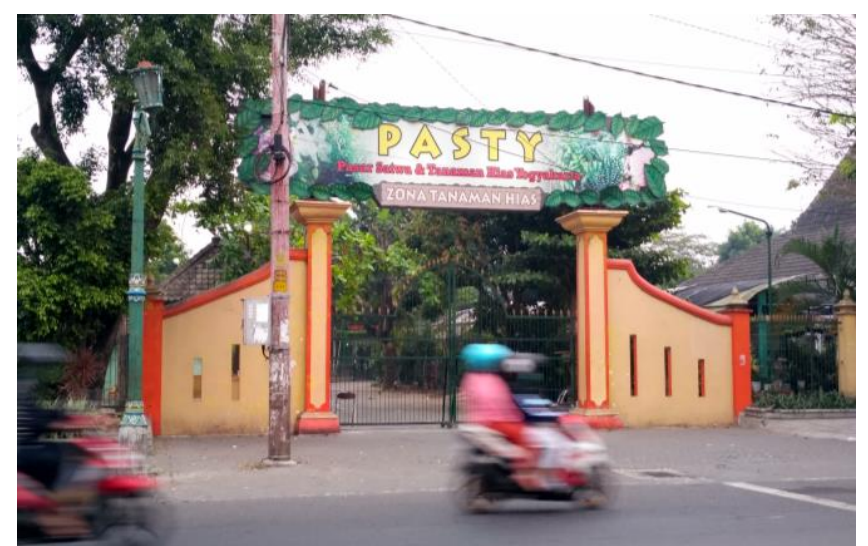

A

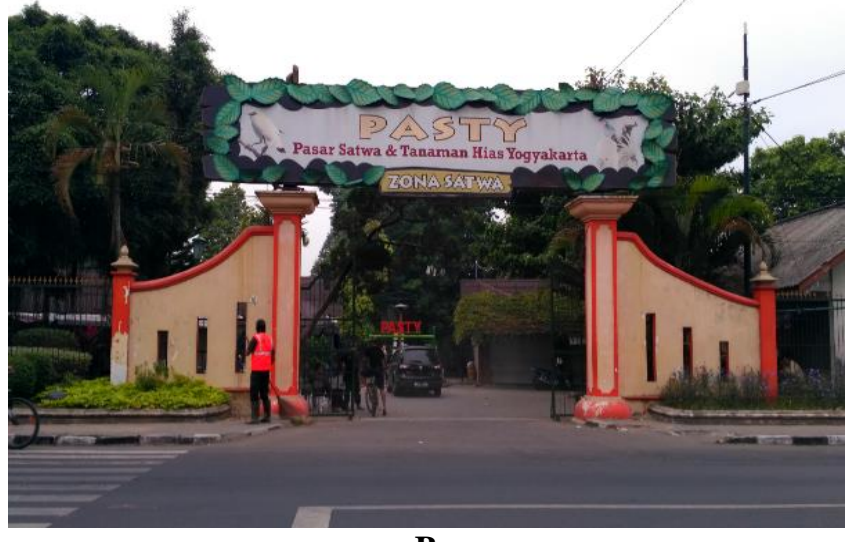

B

Figure 2. The main gate of the traditional market of Pasty, Yogyakarta, consists of 2 main gates namely: A. ornamental plant zone; B. animal zone gate

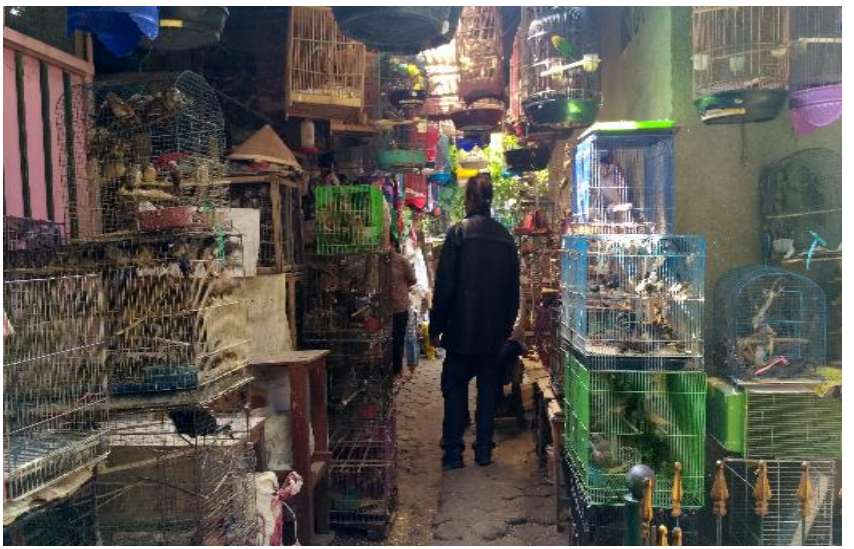

A

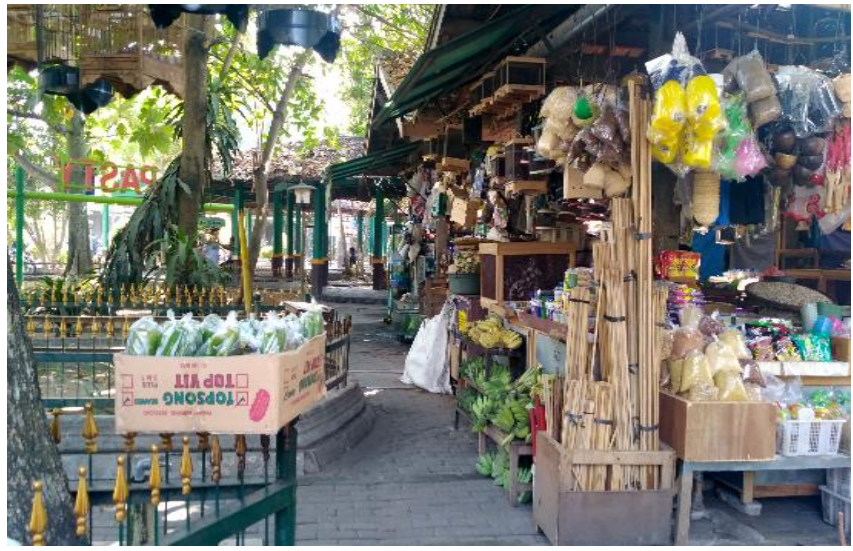

B

Figure 3. A. Birds are put in cages and being placed and hung in the front of stall; B. Various cages; perch; feed; and vitamin and medicine for birds are commonly sold in stalls of the bird market Pasty Yogyakarta

\section{Research procedures}

The method used in this study was qualitative with ethnoornithology approach (Albuquerque et al. 2014; Pam 2017; Iskandar 2018). Ethnoornitology is a branch of ethnozoology emerged from the field of ethnosciences, and seek to understand how the world's different people have perceived and interacted with faunal (avifaunal) resources throughout history (Alves and Souto 2015). The ethnoornithology is focused on the local knowledge (LK) or the traditional ecological knowledge (TEK) of birds, and culturally mediated relationships between communities of people and other animals of their environment. Local people's knowledge begins with animal nomenclature and classification in the local idiom (Hunn 2011).

To collect field data some techniques, including observation, bird surveys, and semi-structure interview were employed. Observation is aimed at the observed general condition of the bird market of Pasty. In terms of observation, the researchers first visited the bird market of
Pasty in the morning, at that time every bird trader started work to open his or her stall (kios) to take out bird cages and hung it in the front of the stall. Observations were employed by both non-participants and participants (Newing et al. 2011). Non-participants were undertaken by researchers observed the general condition of bird market condition and various activities of bird trader and bird buyer activities. While participant observation, the researchers actively involved in some activities of bird traders, including providing bird foods. The field observations were conducted in 2 periods with an interval of one period for the next period of about 6 months. Each period the researcher visits every day to the bird market for a week. Researchers visited bird traders in each stall to introduce themselves and asked for permission to research the bird market that firstly ask the local authority of the market service to ask research permission. This is considered as approvals and participant consent in conducting research. 


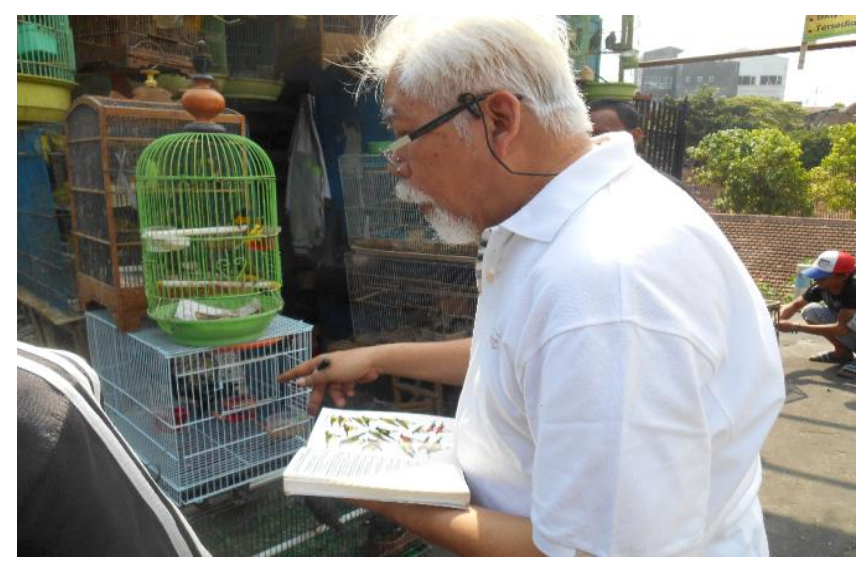

$\mathbf{A}$

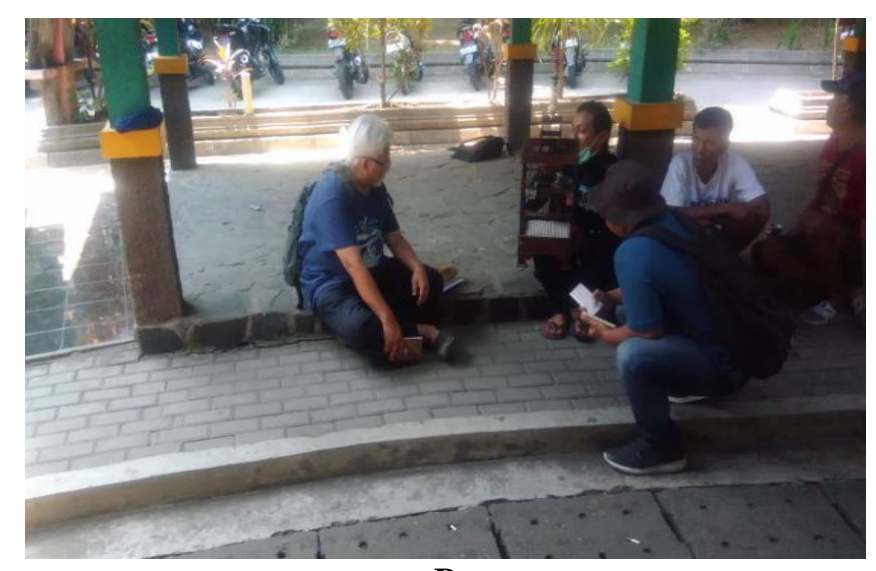

B

Figure 4. A. The researcher is identifying bird species; B. The researcher is interviewing informants at the bird market

The field research intended so that researchers can record the species of birds traded at each stall, and research can interview with traders (cf. Albuquerque et al. 2014 ${ }^{\mathrm{a}, \mathrm{b}}$ ). Moreover, on the second day and following day, and after the traders were familiar with the researchers, a survey of the species of birds traded by each bird trader was carried out (Figure 4). Bird survey was intended to record each species that being traded by bird traders. To identify the species of birds, several books of field guide to the birds of Western and Eastern Indonesia (Papua and New Guinea), including a book written by Hoogerwerf 1949; King and Dickinson 1975; Mackinnon et al. 1992; Pratt and Beehler 2015 were used. As for the bird taxonomy system of Indonesian birds followed the taxonomy system of Sukmantoro et al. (2007).

While semi-structured interview or deep interview was employed with competent important who are purposively selected by using the snowball technique. This technique was used in the intentional selection of informants. To explore the competent informant or local expert as bird traders in the bird trader community of bird market of Pasty, one could choose to work only with local experts representing the set individuals who are socially legitimized and recognized as holders of particular knowledge. From the initial contact with the bird trader community, the first expert was identified, and this expert indicated another expert, and so on, until all the community experts were involved (cf. Alburquerque et al. 2014 ${ }^{\mathrm{a}}$ ). Some informants were purposely selected, namely old-men of bird traders; village middlemen who weekly visited bird market for supplying wild birds and natural bird food; and bird buyers. In semi-structured interviews, informants gave extensive responses to a series of general questions, some of which have been in advance, and some of which arise naturally during the conversation (Figure 4). The time for interviews was selected when the informant was not being busy serving bird buyers and the talks were arranged in an informal and relaxed manner so that the informant was comfortable and did not feel disturbed (Iskandar 2018). The information from the informant during the interview was recorded in the field notebook and was also accompanied by a recording of voice notes of HP.

\section{Data analysis}

Data obtained from observation, bird survey, and semistructured interview that predominant semi-quantitative data and non-numerical data-most commonly either words or pictures were analyzed, namely made critical analysis and construction of a narrative count. The qualitative analysis involved cross-checking, summarizing, and synthesizing from sources to build up a narrative account (Newing et al. 2011).

Cross-checking was undertaken by appropriating check data to validate data, namely researchers used triangulation that is compared to the data of observations and bird surveys with data obtained from interviews. The researcher conducted comparing interview data from one informant to another. Besides, the results of this field study were also analyzed with some report's literature.

\section{RESULTS AND DISCUSSION}

\section{Bird species traded}

Based on direct observation, direct census, and interview with informants of bird traders, 106 bird species, representing 40 families were encountered in the bird market of Pasty (Table 1). As can be seen from Table 1, the bird diversity in the bird market of Pasty can be considered to be high. For example, if it is compared to that of bird market Bogor, West Java (54 species) but is considered lower compared to that of bird market of Sukahaji, Bandung, West Java (128 species), and bird market of Pramuka, East Jakarta (180 species) (Cng et al. 2015; Wiedarti et al. 2016; Iskandar et al. 2019 a).

Although in terms of the bird diversity of bird market of Pasty is high, the species evenness referring to how close individual numbers each species in a bird community in the bird markets is considered to below. For example, based on this study it can be revealed that only $27 \%$ of species 
classified as dominant and subdominant, while $73 \%$ of species classified as non-dominant. Some bird species are classified as dominant and sub-dominant species, such as Melopastacus undulatus, Serinus canaria, Agapornis sp, Columba livia, Geopelia striata, Streptopelia chinensis, Pycnonotus aurigaster, Pycnonotus goiavier, Sturnus contra, Coturnix chinensis, Lonchura maja, Loncura punctulata, Lonchura leucogastroides, and Lonchura manyar (Table 1). Some of the dominant species, including Melopasticus undulatus, Serinus canaria, Agapornis sp, Columba livia, and Coturnix chinensis are categorized as non-wild species that are raised by bird farms instead of hunting from ecosystems.

Among the bird species with a low population in the bird market of Pasty, 14 species are documented as categorized as the protected bird in Indonesia based on Law No.5 of 1990 concerning Conservation of Biological Natural Resources and their Ecosystems, and the Government Regulation No. P.20/ MENLHK/setjen/Kum.1 / 6/208. All species of protected birds i.e. Cacatua galerita, Megalaima corvina, M. javensis, M.lineata, Chloropsis sonnerati, C. cochinchinensis, Cissa thalassina, Platylophus galericulatus, Falco moluccensis, Rhapidura javanica, Pavo muticus, Lorius lory, and Gracula religiosa (Figure 5; Table 1). Formally, those protected birds are prohibited to raise and trade both in the form of a dead specimen without permission from the government (Department of Forestry (2007).

There is no doubt that although some species have been protected by law in Indonesia, those species have been predominantly sold in many bird markets. For example, in addition to Pasty Yogyakarta, some bird protected are also recorded in bird market of Pramuka East Jakarta; Sukahaji Bandung, Bogor bird markets, West Java; and Splendid, Malang, East Java (Janiawati 2012; Chng et al. 2015; Wiedarti et al. 2016; Iskandar et al. 2019).

Therefore, it can be inferred that although some birds in Indonesia have been protected, there is no guarantee that those species can survive life in their natural habitat or ecosystem because illegal hunting and trade in rare and protected bird species continue in many regions (PangauAdam and Noske 2010; Suba et al. 2011; Cng et al 2015; Shepherd and Cassey 2017; Setioko 2019; Hakim et al. 2020). Some socio-economic and cultural factors cause rampant illegal hunting and trade in rare bird species in Indonesia, including hunting and trading of species, may provide economic benefits, control cannot be properly undertaken regularly and sustainably; there is no strict action against 'lawbreaker'; lack of awareness from the community; and conservation programs that do not involve the community. Neglect of local community socio-cultural aspects, including the traditional ecological knowledge (TEK) and the ecological wisdom of the nature conservation, has been realized for a long time by scholars. Many scholars have realized that most of the environmental component changes, including flora and fauna are social -they are to do with people-- and therefore many of the biggest, most urgent challenges facing conservationist are to do with social, economic and political systems, but mainly in past, the people-conservation interface has been neglected (Cunninham 2001; Carlson and Maffi 2004; Newing et al. 2011; Pam 2017). Therefore, an urgent need to develop social science experience within the broad discipline of conservation (Newing et al. 2011). Ethnoornithology as a trans discipline has evolved over the past several decades bringing together nature conservation and the TEK system in ecological management and biodiversity conservation (Pam 2017).

\section{Naming and bird classification}

The bird traders of the Pasty market are Javanese ethnic. Based on Javanese terms for birds generally is manuk or in the Indonesian language called as burung. Most birds traded at the Pasty bird market have a common local name or vernacular name and birds can be classified based on vocalization; color; morphological characteristics; habitat, distinctive behavior; flying racing competition; involving of song competition; selling price; and wilderness (Table 2).

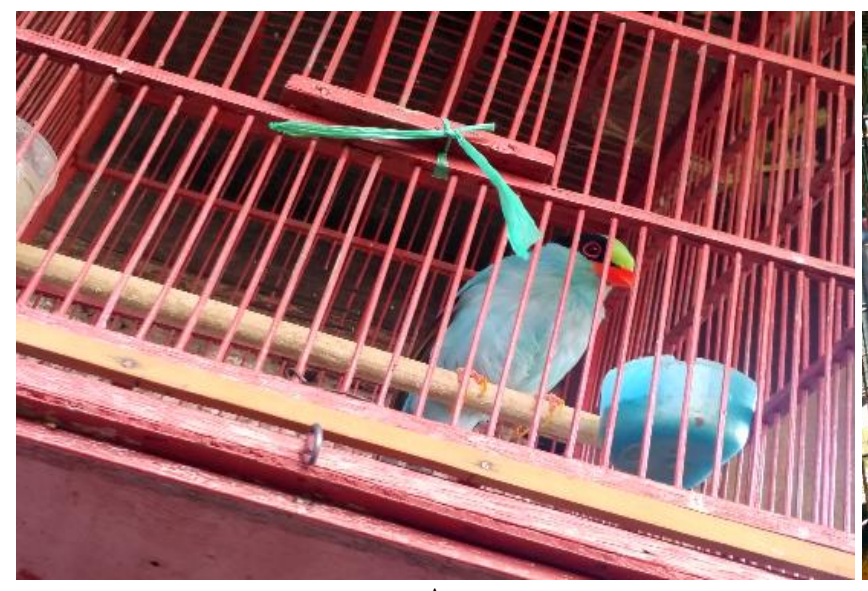

A

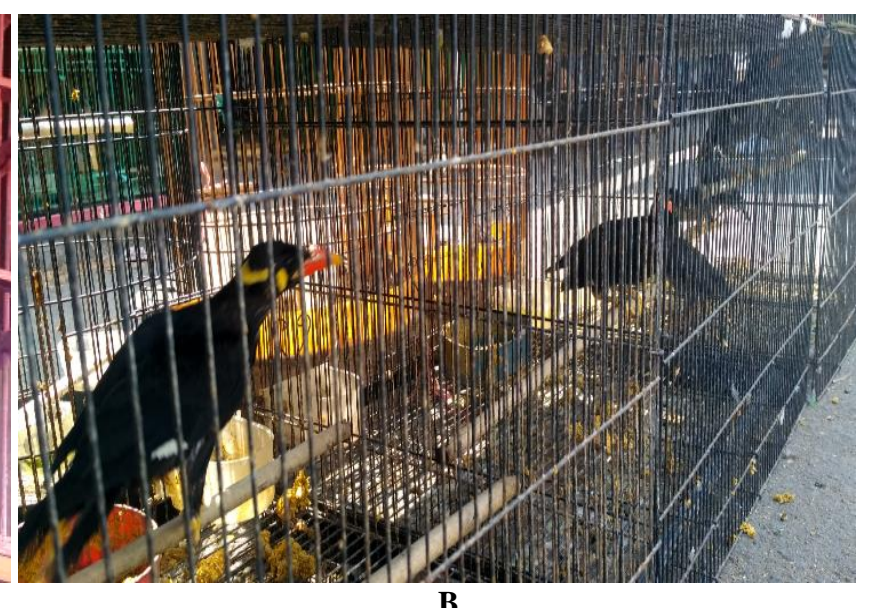

B

Figure 5. A. Bird of Ekek keling -Javan Green Magpie- (Cissa thalassina) and; B. Beo -Hill Myna- (Gracula religiosa) as protected bird in Indonesia are being traded in the bird market 
Table 1. Various birds were encountered in the bird market of Pasty, Yogyakarta, Indonesia based on conducted the field research August 2019 and February 2020

\begin{tabular}{|c|c|c|c|c|c|c|}
\hline Family & Species & Vernacular name & Indonesia name & Common name & Selling price (Rupiah) & Population $* *)$ \\
\hline Aegithinidae & Aegithina tiphia & Cipo,sirtu & Cipoh kacat & Common Iora & $250,000-300,000$ & Non- dominant \\
\hline Alaudidae & Mirafra javanica & Branjangan & Brnajangan jawa & Horsfield's bush lark & $125,000-250,000$ & Sub-dominant \\
\hline \multirow[t]{2}{*}{ Alcedinidae } & Halcyon cyanoventris & Cucak urang & Cekakak Jawa & Javan kingfisher & $80,000-250,000$ & Non- dominant \\
\hline & Todirhampus (Halcyon-chloris) & Cucak urang & Cekakak-sungai & Collared kingfisher & $125,000-250,000$ & Non-dominant \\
\hline Anatidae & Dendrocygna acuta & Meliwis & Belibis kembang & Lesser whistling-duck & $90,000-150,000$ & Non-dominant \\
\hline Artamidae & Artamus leucorynchus & Dedet & Kekeb Babi & $\begin{array}{l}\text { White-breasted Wood- } \\
\text { swallow }\end{array}$ & $100,000-200,000$ & Non-dominant \\
\hline Cacatuidae & Cacatua galerita*) & Kakatua Jambul kuning & Kakatua koki & Sulphur-Crested Cockatoo & $2,500,000-3,000,000$ & Non-dominant \\
\hline \multirow[t]{4}{*}{ Campephagidae } & Hemipus hirundinaceus & Jing-jing teureup & Jing-jing bukit & $\begin{array}{l}\text { Black-winged Flycatcher } \\
\text { shrike }\end{array}$ & $250,000-400,000$ & Non-dominant \\
\hline & Lalage nigra & Kapasan & Kapasan kemiri & Pied Triller & $150,000-300,000$ & Non-dominant \\
\hline & Pericrocotus cinnamomeus & Mantenan & Sepah kecil & Small minivet & $500,000-800,000$ & Non-dominant \\
\hline & Pericrotus flammeus & Mantenan & Sepah hutan & Scarlet Minivet & $500,000-800,000$ & Non-dominant \\
\hline \multirow{4}{*}{ Capitonidae } & Megalaema*) corvina & Kutut gung & Takur bututut & Brown-throated Barbet & $25,000-50,000$ & Non-dominant \\
\hline & Megalaema haemacephala & Engkuk & Takur engkut-engkut & Coppersmith & $200,000-350,000$ & Non-dominant \\
\hline & Megalaema javensis*) & Trontong & Takur tulung tumpuk & Black-banded Barbet & $200,000-350,000$ & Non-dominant \\
\hline & Megalaima lineata*) & Glotok & Takur bultok & Lineated Barbet & $200,000-350,000$ & Non-dominant \\
\hline \multirow{2}{*}{ Chloropseidae } & Chloropsis sonnerati*) & Cucak ijo, Cucak meranti & Cica daun besar & Greater Green Leafbird & $200,000-350,000$ & Non-dominant \\
\hline & Choloros cochinchinensis *) & Cucak ijo & Cica daun sayap biru & Blue-winged Leafbird & $200,000-350,000$ & Sub-dominant \\
\hline \multirow[t]{6}{*}{ Columbidae } & Chalcopaps idica & Derkuku lumut & Delimukan zamrud & Emerald dove & $25,000-50,000$ & Non-dominant \\
\hline & Columba livia & Merpati, Doro & Merpati & Rock dove, Feral pigeon & $75,000-200,000$ & Dominant \\
\hline & Geopelia striata & Kutut, Perkutut & Perkutut jawa & Zebra dove, Peaceful dove & $25,000-250,000$ & Dominant \\
\hline & Streptopelia bitorquata & Puter & Dederuk jawa & Sunda collared dove & $30,000-300,000$ & Dominant \\
\hline & Streptopelia chinensis & Deruk & Tekukur biasa & Spootted dove & $30,000-300,000$ & Dominant \\
\hline & Treron vernans & Walik & Punai gading & Pink-necked green-pigeon & $400,000-500,000$ & Non-dominant \\
\hline \multirow[t]{4}{*}{ Corvidae } & Cissa thalassina*) & Ekek keling & Ekek geling & Javan Green Magpie & $150,000-400,000$ & Non-dominant \\
\hline & Corvus enca & Engkak & Gagak hutan & Splender-billed crow & $100,000-300,000$ & Non-dominant \\
\hline & Corvus macrorhynchos & Gaok & Gagak kampung & Large-billed crow & $100,000-300,000$ & Non-dominant \\
\hline & Platylophus galericulatus*) & Cililin & Tangkar ongklet & Crested shrike-jay & $75,000-200,000$ & Non-dominant \\
\hline \multirow[t]{3}{*}{ Cuculidae } & Cacomantis merulinus & Wikwik & Wikwik lurik & Plantive Cockatoo & $100,000-200,000$ & Non-dominant \\
\hline & Eudynamys scolopaceus & $\begin{array}{l}\text { Culik-culik (male), Tuha } \\
\text { (female) }\end{array}$ & Tuwur Asia & Asian koel & $1,500-2,000,000$ & Non-dominant \\
\hline & Ketapa ketupu & Kutuk-kutuk & Beluk ketupa & Buffy-fish owl & $20,000-300,000$ & Non-dominant \\
\hline \multirow[t]{3}{*}{ Decidae } & Dicaeum sanguilentum & Burung cabai & Cabai gunung & $\begin{array}{l}\text { Blood-breasted flower } \\
\text { pecker }\end{array}$ & $100,000-200,000$ & Non-dominant \\
\hline & Dicaeum trigonostigma & Burung cabai & Cabai bunga-api & Orange-bellied & $100,000-200,000$ & Non-dominant \\
\hline & Dicaeum trochileum & Manuk/Burung cabai & Cabai jawa & $\begin{array}{l}\text { Scarlet-headed } \\
\text { Flowerpecker }\end{array}$ & $100,000-200,000$ & Non-dominant \\
\hline
\end{tabular}


Emberizidae

Estrildidae

Emriza sp.

Erythrina prasinia

Lonchura feruginosa

Lonchura leucogastroides

Lonchura maja

Lonchura punctulata

Padda oryzivora

Falconidae

Fringillidae

Laniindae

Melagrinidae

Falco moluccensis*)

Serinus canaria

Lanius schach

Muscicapidae

gris domesticus

yornis banyumas

Ficidula mugimaki

Saxicola caprata

Rhipidurae

Nectariniidae

Rhipidura javanica *)

Aethopyga sp.

Cinnyris (Nectarinia) jugularis

Leptocoma (Nectaria) separata

Phasianidae

Arborophila javanica

Coturnix chinensis

Gallus sp.

Gallus domesticus

Pavo muticus*)

Orioidae

Paridae

Picidae

Oriolus chinensi

Parus major

Chrysocolaptes lucidus

Dendrocopos macei

Dinopium javanense

Pitta guajana*)

Ploceidae

Passer montanus

Ploceus manyar

Ploceus philippinus

Psittacidae

Loriculus lory*

Melopsittacus undulatus

Psittacula alexandri

Srigunting

Srigunting kantil

Emprit Jepang

Bondol ijo

Emprit haji

Emprit

Emprit haji

Emprit peking

Gelatik

Alap-alap

Kenari

Pentet, Cendet

Kalkun

Sulingan, Selendang biru

Srikatan

Decu

Srikatan

Kolibri

Sogok ontong, sontong

Kolibri

Puyuh gonggong

Puyuh telur

Ayam mutiara

Ayam kate

Merak

Podang

Gelatik wingko

Platuk bawang

Platuk semut

Platuk kundang

Anis pancawarna, Paok

Gereja

Manyar

Manyar

Lovebird

Nuri Irian

Parkit, Palek, Kalek

Betet
Srigunting hitam

Srigunting bukit

Pipit Jepang

Bondol hijau binglis

Bondol oto-hitam

Bondol jawa

Bondol haji

Bondol peking

Gelatik jawa

Alap-alap sapi

Kenari

Bentet kelabu

Kalkun

Sikatan cacing

Sikatan mugimaki

Decu belang

Kipasan belang

Burung madu

Burung madu sriganti

Burung madu

pengantin

Puyuh gongong jawa

Puyuh batu

Ayam mutiara

Ayam kate

Merak hijau

Kepudang kuduk hitan

Gelatik batu kelabu

Platuk tunggir-emas

Caladi ulam

Platuk besi

Paok pancawarna

Burung gereja Erasia

Manyar jambul

Manyar tempua

Lovebird

Kasturi Kepala Hitam

Parkit

Betet biasa
Black drongo

The Lesser racket-tailed

drongo

Bunting

Pun-tailed Parrot finch

Chestnut munia

Javan munia

White-headed munia

Scaly-breasted munia

Java sparrow

Spotted kestrel

Island canary

Long-tailed shrike

Wild turkey

Hill Blue-flycatcher

Mugimaki flycatcher

Pied bush chat

Pied bush chat

Sunbird

Olive-backed sunbird

Purple-throated sunbird

Chestnut-bellied partridge

Slender-billed crow

Guinea pow

Bantam chicken

Green peafowl

Black-naped oriole

Great tit

Greater flame back

Fulvous breasted

Common goldenback

Bandel pitta

Europian tree sparrow, tree

sparrow

Streaked weaver

Baya weave

Lovebird

Black-capped lory

Budgerigar

Red-breasted parakeet
150,000-200,000

$200,000-250,000$

Non-dominan

Non-dominant

200,000-350,000

$1,000-3,000$

$1,000-3,000$

$1,000-2,000$

$5,000-7,500$

$1,000-2,000$

$150,000-300,000$

$350,000-400,000$

$150,000-400,000$

$85,000-250,000$

$80,000-200,000$

$350,000-2000,000$

$100,000-200,000$

$100,000-450,000$

$100,000-450,000$

250,000-350,000

200,000-500,000

$200,000-500,000$

150,000-300,000

$7,000-125,000$

$300,000-450,000$

$100,000-300,000$

$200,000-400,000$

$300,000-350,000$

$125,000-400,000$

$125,000-350,000$

$100,000-250,000$

$125,000-400,000$

$125,000-400,000$

$1,000-2,500$

$35,000-150,000$

$35,000-125,000$

150,000-650,000

$700,000-900,000$

$100,000-200,000$

250,000-300,000
Non-dominan

Non-dominant

Dominant

Dominant

Dominan

Dominant

Sub-dominant

Non-Dominan

Dominant

Sub-dominant

Non-dominant

Non-dominant

Non-dominant

Non-dominant

Non-dominant

Non-dominant

Non-dominan

Non-dominant

Non-Dominan

Dominant

Non-dominant

Non-dominant

Non-dominant

Non-dominan

Sub-dominant

Non-dominant

Non-dominant

Non-dominant

Non-dominan

Non-dominant

Dominant

Dominan

Dominant

Non-dominant

Dominant

Non-dominant 


\begin{tabular}{|c|c|c|c|c|c|c|}
\hline \multirow[t]{8}{*}{ Pycnonotidae } & Criniger (Alophoxius) bres & Cucak jenggot & Empuloh janggut & Grey-cheeked Bulbul & $100,000-300,000$ & Non-dominant \\
\hline & Ixos (Iole) virescens & Brinjil gunung & Brinjil gunung & Sunda bulbul & $100,000-400,000$ & Non-dominant \\
\hline & Pycnonotus atriceps & Tilan sutro & Cucak kuricang & Black-headed bulbul & $85,000-100,000$ & Non-dominant \\
\hline & Pycnonotus aurigaster & Tilan, Gentilang & Cucak kutilang & Sooty-headed bulbul & $7,000-50,000$ & Dominant \\
\hline & Pycnonotus bimaculatus & Cucak rantai & Cucak gunung & Orange-spotted bulbul & $80,000-90,000$ & Non-dominant \\
\hline & Pycnonotus goivier & Cerocokan & Merabah cerucuk & Yellow-vented bulbulu & $35,000-750,000$ & Dominant \\
\hline & Pycnonotus melanicterus & Tilang mas & Cucak kuning & Black-crested bulbul & $80,000-100,000$ & Non-dominant \\
\hline & Pycnonotus zeylanicus & Cucak rowo & Cucak rawa & Straw-headed bulbul & $850,000-15,000,000$ & Non-dominant \\
\hline Sittidae & Sitta frontalis & Rambatan & Munguk beledu & Velvet-fronted nuthatch & $150,000-450,000$ & Non-dominant \\
\hline Strigidae & Otus lempiji & Bueuk & Celepuk reban & Sunda-scop-owl & $75,000-100,000$ & Sub-dominant \\
\hline \multirow[t]{7}{*}{ Sturnidae } & Acridotheres javanicus & Jalak kebo & Jalak kerbau & White-vented myna & $85,000-125,000$ & Dominant \\
\hline & Acridotheres tristis & Kerak ungu & Kerak ungu & Common myna & $85,000-150,000$ & Non-dominant \\
\hline & Aplonis minor & Jalak keling & Perling kecil & Short-tailed Starling & $100,000-800,000$ & Non-dominant \\
\hline & Gracula religiosa* & Beo & Tiong mas & Hill myna & $400,000-900,000$ & Non-dominant \\
\hline & Leucopsar rothschildi*) & Jalak putih Bali & Curik Bali & Bali Myna & $10,000,000-25,000,000$ & Non-dominant \\
\hline & Sturnus contra & Jalak uren & Jalak suren & Asian pied starling & $400,000-700,000$ & Dominant \\
\hline & Sturnus melanpoterus*) & Jalak puteh & Jalak putuh & Black-winged starling & $450,000-750,000$ & Non-dominant \\
\hline \multirow[t]{4}{*}{ Sylviidae } & Orthotomus ruficeps & Prinjak & Cinenen kelabu & Ashy tailorbird & $65,000-300,000$ & Sub-dominant \\
\hline & Orthotomus sutorius & Prinjak lumut & Cinenen pisang & Common tailor bird & $65,000-300,000$ & Sub-dominant \\
\hline & Phylloscopus trivirgatus & Cikrak & Cikrak daun & Mountain leaf-warbler & $100,000-200,000$ & Non-dominant \\
\hline & Prinia familiaris & Ciblek & Perenjek jawa & Bar-winged prinia & $75,000-700,000$ & Sub-dominant \\
\hline \multirow[t]{3}{*}{ Timaliidae } & Garrulax chinensis & Poksay & Poksay hitam & $\begin{array}{l}\text { Back-throated laughing } \\
\text { thrush }\end{array}$ & $350,000-2,000,000$ & Non-dominant \\
\hline & Garrulax leucolophus & Poksay Sumatra & Poksay jambul & White-crested laughthrush & $500,000-1,000,000$ & Non-dominant \\
\hline & Pomaturhinus montanus & Bongkrek, Kopi-kopi & Cicakopi melayu & $\begin{array}{l}\text { Chestnut-backed scimitar } \\
\text { babbler }\end{array}$ & $150,000-400,000$ & Non-dominant \\
\hline Turnicidae & Turnix suscitator & Gemak Cpuyuh & Gemak loreng & Red-backed buttonquail & $100,000-250,000$ & Sub-dominant \\
\hline \multirow[t]{5}{*}{ Turdidae } & Bracypterix montana & Cingcoang & Cingcoang biru & Javan shortwing & $150,000-200,000$ & Sub-dominant \\
\hline & Copsychus malabaricus & Larwo & Kucica hutan & White-rumped Shama & $800,000-15,000,000$ & Sub-dominant \\
\hline & Copsychus saularis & Kacer & Kucica kampung & Oriental magpie robin & $300,000-1,200,000$ & Sub-dominant \\
\hline & Zoothera citrina & Anis merah, Punglor & Anis merah & Orange-headed thrush & $350,000-3,000,000$ & Sub-dominant \\
\hline & Zoothera (Geokichla) interpres & Anis kembang & Anis kembang & Chestnut-capped thrush & $700,000-5000,000$ & Sub-dominant \\
\hline \multirow[t]{2}{*}{ Tytonidae } & Phodius badius & Serak & Serak bukit & Oriental bay-owl & $100,000-250,000$ & Non-dominant \\
\hline & Tyto alba & Gares & Serak Jawa & Barn owl & $300,000-350,000$ & Non-dominant \\
\hline \multirow[t]{3}{*}{ Zosteropidae } & Zosterops chloris & Kacamata, Pleci & Kacamata laut & Lemon-bellied white eye & $45,000-250,000$ & Dominant \\
\hline & Zosterops montanus & Kacamata, Pleci & Kacamata gunung & Montain white-eye & $45,000-250,000$ & Dominant \\
\hline & Zosterops palpebrosus & Kacamata, Pleci & Kacamata biasa & Oriental white eye & $45,000-250,000$ & Dominant \\
\hline
\end{tabular}

MENLHK/sateen/Kum.1 / 6/208. **) Dominant > 20 individuals ; Sub-dominant 11-20 individual ; Not-dominant 1-10 individuals 
Table 2. Folk classification of birds based on the bird traders

\begin{tabular}{|c|c|c|c|c|}
\hline Folk classification & Vernacular name & Scientific name & English name & Bird trader perception \\
\hline \multirow{17}{*}{$\begin{array}{l}\text { Distinctive } \\
\text { /vocalization }\end{array}$} & Cipo & Aegithina tiphia & Common iora & Voice: cipo-cipo-cipo \\
\hline & Bubut & Centropus bengalensis & Lesser coucal & Voice: bubut-bubut-bubut \\
\hline & Cingcoang & Brachypterix montana & Javan shortwing & Voice: cingcoang-cingcoang-cingcoang \\
\hline & Ekek keeling & Cissa thalassina & Javan green magpie & Voice: ekekkeling-ekekkeling-ekekkeling \\
\hline & Engkak & Corvus enca & Slender-billed crow & Voice:engkak-engkak-engkak \\
\hline & Gaok & Corvus macrorhynchos & Large-billed crow & Voice:gaok-gaok-gaok \\
\hline & Kututut, Perkutut & Geopelia striata & $\begin{array}{l}\text { Zebra-dove; } \quad \text { Peaceful } \\
\text { dove }\end{array}$ & Voice: kututut-kututut-kututut or perkutut-perkutut-perkutut-perkutut \\
\hline & Kutuk-kutuk & Кетири кетири & Buffy-fish-owl & Voice: kutukkutuk-kutukkutuk-kutukkutuk \\
\hline & Emprit & Lonchura leucogastroides & Javan munia & Voice: emprit-emprit-emprit \\
\hline & Emprit peking & Lonchura punctulata & Scaly-breasted Munia & Voice: peking-peking-peking \\
\hline & Engkuk engkuk & Megalaema haemacephala & Coppersmith barber & Voice:engkuk-engkuk-engkuk \\
\hline & Trontong & Megalaema javensis & Black-banded barbet & Voice:trontong-trontong-trontong \\
\hline & Kluto, Glotok & Megalaema lineata & Lineated barbet & Voice:kluto-kluto-kluto or glotok-glotok-glotok \\
\hline & Ciblek & Prinia familiaris & Bar-winged prinia & Voice:ciblek-ciblek-ciblek \\
\hline & Corokan & Pycnonotus goiavier & Yellow-vented Bulbul & Voice: corok-corocok-corocok \\
\hline & Tilan, Gentilang & Pycnonotus aurigaster & Sooty-headed Bulbul & Voice: tilan-tilan-tilan or gentilang-gentilang-gentilang \\
\hline & Culik-culik & Eudynamis scolopaceus (male) & Common coel & Voice: culik-culik-culik \\
\hline \multirow[t]{12}{*}{ Special color } & Jalak keeling & Aplonis minor & Short-tailed starling & Mature birds have shiny black feathers (keling) and red eyes \\
\hline & Derkuku lumut & Chalcopaps indica & Emerarl dove & Bird has a dark metallic green-blue resembling moss (lumut) \\
\hline & Slendang biru & Cyornis banyumas & Hill blue-flycatcher & Bird has shining blue on upper parts is likened to a blue shawl (selendang biru) \\
\hline & Kerak ungu & Acridotheres tristis & Common mayna & It has a brown bluish body (ungu) \\
\hline & Burung cabe & Dicaeum trochileum & $\begin{array}{l}\text { Scarlet-headed } \\
\text { Flowerpecker }\end{array}$ & Adult male birds on the head, back, stump and chest have a red-colored scar \\
\hline & Bondol ijo & Erythrina prasina & Pin-tailed parrotfinch & $\begin{array}{l}\text { The male bird has green (ijo) upperpart, female-like male but the side of the head } \\
\text { green. }\end{array}$ \\
\hline & Emprit haji & Lonchura maja & White-headed munia & The adult bird has a white head associated with white Hajj skullcap \\
\hline & Prienjak lumut & Orthotomus sutorius & Common tailor bird & Prienjak bird that has general feather color greenish resembling moss color. \\
\hline & Anis pancawarna & Pitta guajana & Banded pitta & $\begin{array}{l}\text { The male bird of anis that has five colors (panca warna), namely black head with } \\
\text { broad yellow eyebrows becoming flaming orange nape, chestnut-brown brown } \\
\text { upperparts with a blue tail and upper tail coverts and white streak on wing coverts }\end{array}$ \\
\hline & Montanan & Pericrocotus cinnamomeus & Small Minivet & $\begin{array}{l}\text { The male bird has a grey head and mantle; and orange-yellow belly that has a } \\
\text { festive red color like a wedding dress (mantenan) }\end{array}$ \\
\hline & Jalak puteh & Sturnus melanopterus & Black-winged Starling & Male bird all white feathers (puteh), except black wings and tail. \\
\hline & Anis bata & Zoothera citrina & Orange-headed Thrush & $\begin{array}{l}\text { The male bird has an orange-rufous head, blue-grey upperparts. Based on the local } \\
\text { people's perception orange-rufous color head is similar to that brick color (warna } \\
\text { bata merah). }\end{array}$ \\
\hline
\end{tabular}




\begin{tabular}{|c|c|c|c|c|}
\hline \multirow[t]{7}{*}{$\begin{array}{l}\text { Morphological } \\
\text { characteristic }\end{array}$} & Cucak jenggot & Alophoixus bres & Grey-cheeked bulbul & $\begin{array}{l}\text { This bulbul bird has yellowish tinge on breast and white chin that is considered as } \\
\text { a bear by local people. }\end{array}$ \\
\hline & Larwo & Cospsychus malabaricus & White-rumped shama & $\begin{array}{l}\text { Larwo comes from the words lare and dowo, meaning lare is feather (bulu), while } \\
\text { dowo means ekor (tail). So, this bird species is perceived by local people to have a } \\
\text { long tail. }\end{array}$ \\
\hline & Platuk semut & Dendrocopos macei & $\begin{array}{l}\text { Fulvous-breasted } \\
\text { Woodpecker }\end{array}$ & Woodpecker has a small body that interpreted as an ant (semut). \\
\hline & Srigunting & Dicrurus macrocerceus & Black Drongo & Bird has tail feathers to be forked to resemble a scissor (gunting) \\
\hline & Srigunting kantil & Dicrurus remifer & $\begin{array}{l}\text { The Lesser Racket-tailed } \\
\text { Drongo }\end{array}$ & Kurantil means to hang, this bird has long tail hanging. \\
\hline & Ayam kate & Gallus domesticus & Bantam chicken & Chicken has a short body (kate) \\
\hline & Burung kaca mata & Zosterops palpebrosa & Oriental White-eye & $\begin{array}{l}\text { Birds with diagnostic signs that their eyes are white like the shape of glasses } \\
\text { (kacamata) }\end{array}$ \\
\hline \multirow[t]{4}{*}{ Distinctive behavior } & Jalak kebo & Acridotheres javanicus & White-vented myna & This bird often looks for food on the back of the buffalo (punggung kebo). \\
\hline & Burung madu & Anthreptes sp & Sunbird & This bird often looks for food on the flower to find flower honey and insects. \\
\hline & Cucak urang & Halcyon cyanoventris & Javan kingfisher & This bird likes to find foods, as shrimp (udang) in an aquatic ecosystem. \\
\hline & Rambatan & Sitta frontalis & Velvet-fronted Nuthatch & $\begin{array}{l}\text { This bird has distinctive behavior to find food by creeping (rambatan) on tree } \\
\text { trunks and branches. }\end{array}$ \\
\hline \multirow[t]{3}{*}{ Special habitat } & Meliwis, meliwis air & Dendrocygna acuta & Lesser Whistling-duck & This bird has a special habitat in various freshwater ecosystems (perairaran). \\
\hline & Prienjak kampung & Orthotomus ruficeps & Ashy tailor & This bird is commonly found in the hamlet area (kampung). \\
\hline & Walik (alas) & Treron vernans & $\begin{array}{l}\text { Pink-necked Green- } \\
\text { pigeon }\end{array}$ & This bird is commonly found in forest area (alas) \\
\hline \multirow{2}{*}{$\begin{array}{l}\text { Flying racing } \\
\text { competition }\end{array}$} & Merpati, Doro-biasa & Columba livia & Rock dove, Feral pigeon & Rock dove is usually not used for flying racing competition (merpati biasa) \\
\hline & Merpati, doro-balap & Columba livia & Rock dove, Feral pigeon & Rock dove is usually used for flying racing competition (merpati balap) \\
\hline \multirow{4}{*}{$\begin{array}{l}\text { Involving in song bird } \\
\text { competition }\end{array}$} & Anis bata & Zoothera citrina & - & Bird song contest \\
\hline & Larwo & Copsychus malabaricus & - & Bird song contest \\
\hline & Kacer & Copsychus saularis & - & Bird song contest \\
\hline & Pleci/Kacamata & - & - & Bird song contest \\
\hline \multirow[t]{10}{*}{ Selling price } & Cucok rowo & - & - & High selling price \\
\hline & - & Copsychus malabaricus & - & High selling price \\
\hline & - & Gracula religiosa & - & High selling price \\
\hline & - & Zoothera citrina & - & High selling price \\
\hline & Poksay & - & - & High selling price \\
\hline & Emprit & - & - & Low selling price \\
\hline & Emprit peking & - & - & Low selling price \\
\hline & Gereja & - & - & Low selling price \\
\hline & Tilan & - & - & Low selling price \\
\hline & Cerocokan & - & - & Low selling price \\
\hline \multirow[t]{2}{*}{ Wilderness } & \multirow{2}{*}{\multicolumn{3}{|c|}{$\begin{array}{l}\text { All species have been just caught from wild (burung bakal) } \\
\text { All species have been tamed or domesticated (burung jadi) }\end{array}$}} & Birds considered to be wild or semi-wild that are known as burung bakal \\
\hline & & & & $\begin{array}{l}\text { Birds have been tamed as a catch from the wild or captivity are locally called as } \\
\text { burung jadi }\end{array}$ \\
\hline
\end{tabular}




\section{Bird vocalization}

Various bird species traded in the bird market of Pasty are well known by bird traders and are locally classified by informants (folk classification) based on bird vocalization. For example, some bird species, including Aegithina typhia, Centropus bengalensis, Geopelia striata, Pycnonotus goiavier, Lonchura leucogastroides, and Megalema haemacephala are locally recognized have common local names as cipo, bubut, kututut, cerocokan, emprit, and engkuk-engkuk, respectively. All these common local names are based on their voices are traditionally heard according to Javanese local people (Table 2).

On the basis the bird traders perception on local bird names, it is generally similar to that of Hoogerwerf (1949) in that some birds, including Aegithina tiphia, Centropus bengalensis, Geopelia striata, Pycnonotus goiavier, Lonchura leucogastroides, and Megalaema haemacephala are recognized own common local name as tipo, cipo or cipeu; dudut troktrok; perkutut; jokjok or cerucuk; piit, pipit and emprit; and engkut or ungkut-ungkut, respectively, are named based on their vocalization.

\section{Special color}

Based on the informants of bird traders of the Pasty bird market, some birds are known to have a common local name based on the feather color of birds. For example, Orthotomus sutorius has a local name as prenjak lumut or green like moss (hijau seperti lumut). Pitta guajana has a local name as anis pancawarna. Panca warna means five colors. So the local name of this bird recognized as panca warna (five colors) because this bird has five colors of feathers, i.e. black color on the head, yellow on eyebrows, brown on back and wing, white color in the form of wing lines and on the chin; and blue color in on tail.

Another bird, Sturnus melanopterus is culturally recognized as a common local name as jalak puteh or jalak putih that jalak means bird of 'starling' and puteh or putih is white color. Because this bird species has a general color of the feather is white, such as the head, neck, back, chest, and stomach are predominant white color. Meanwhile on the wing cover has a gray color, and around the eyes and feet has a yellow color. Pericrocotus cinnamomeus has a local name as mantenan. This is because according to the bird traders this bird has colorful colors that it is similar to that of wedding decoration. The male bird, for example, has a gray head and mantle, orange-yellow belly, while the female has orange rump and dusky white breast. Zoothera citrina is recognized by a bird trader that has close relation with the color of the feather of this bird, as 'anis bata'. Anis is a general name for birds of genera Zoothera, and 'anis bata' means kind of anis' bird that has a color similar to that of brick or orange-rufous. This male bird has orangrufous head, and blue-grey upperparts. In addition, Dicaceum trochileum has a common local name as burung cabe that is to translate literally as a red-pepper bird. This is because of the male orange redback, while the female has red on the ridge, head, and mantle, and belly dirty white.

\section{Morphological characteristics}

Some bird species have also various common local names that are based on distinctive morphological characteristics. For example, Alophoixus bres is locally known as cucak jenggot, because this bird has a characteristic i.e. gray side of head; more olive upperparts that contrast with a rufous-brown upper tail; yellowish tine on the breast (janggut); and pale yellow belly.

Copsychus malabaricus is locally known as larwo. Larwo comes from the words 'larwe' and 'dowo' meaning lare (feather) and dowo means ekor (tail) with a long feather. This bird has characteristics, i.e. male has a black head, throat, upper breast, and upper part, with white rump, long tail with upper tail coverts and outer tail feathers, and dark orange-rufous underpart distinctive.

Dicrurus macrocercus is local commonly known as 'srigunting' because this bird that has feather colors are all black, their tails are very long to wag or fork to form like 'scissors'. Meanwhile, Dicrurus remifer is locally known as Kantil Srigunting. In the Javanese local language, krantil means to hang, so this long tail is like hanging. Morphologically, this bird has shiny black feathers with a racket at the end, like hanging. Therefore, the common local name of the bird is known as srigunting krantil. Meanwhile, Lonchura maja has a common local name as Emprit Haji because the head of this bird has a white color, associated with Hajj Skullcap (Figure 6).

\section{Special habitat}

Some bird species that are traded in the bird market of Pasty are also known to have a common local name reflecting the habits of life in different habitats. For example, Dendrocygna auta is known to have the local name as meliwis or melwis air. Because this bird has the main habitat in some freshwater ecosystems (air), including swamp, lake, paddy field, and others. They usually look for various foods, some organisms in the water, by diving repeatedly while continuing to swim in the freshwaters.

Several species tailorbirds, such as Orthotomus ruficeps and Orthotomus sutorius are locally known in general name as manuk prinjak or 'prinjak bird'. Orthotomus ruficeps, however, is known to own a specific local name as prenjak kampung. This because this bird is predominantly found in the village or hamlet area (kampung). Conversely, Treron vernans is usually called by the local community as walik or walik alas. Because this bird has a habitat in the forest, the common local name is popularly called as walik alas or walik of forest (alas).

Perception of the local community (emic) is similar in that it is based on ecological western scientific knowledge or ornithologist data (ethic), this bird usually looks after foods, including various fruits trees of Ficus spp in pairs or small group in the forest (King and Dickinson 1975; Mackinnon et al. 1992). 


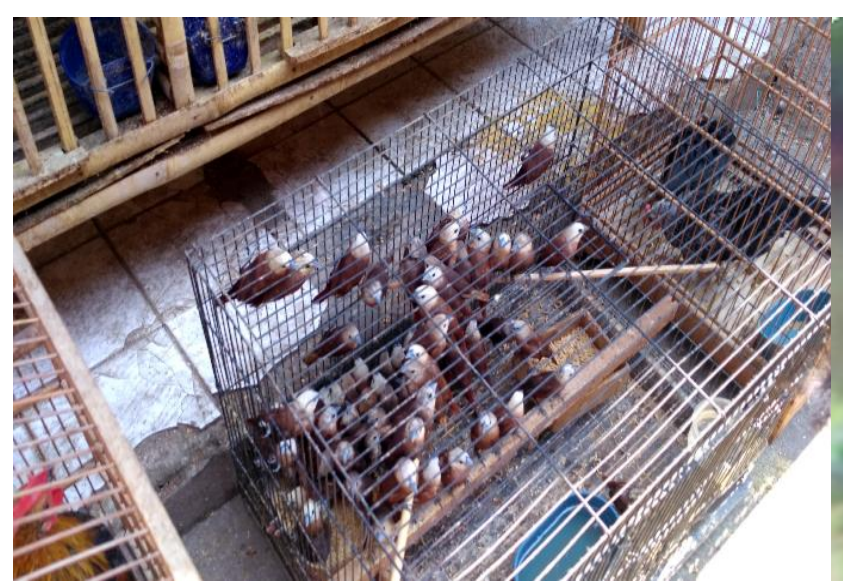

A

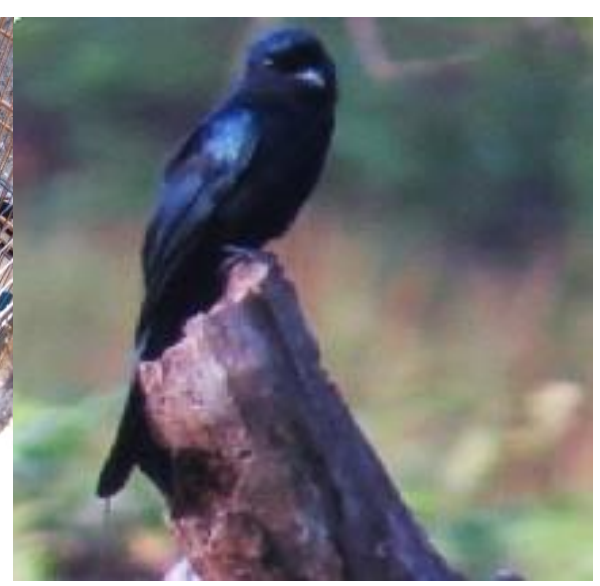

B

Figure 6. The bird which is vernacularly named as emprit haji (Lonchura maja) because of the white head has an association with hajj skullcap (A) and srigunting kantil (Dicriurus remifer) because the tail is long hanging (B)

\section{Distinctive behavior}

Some bird species are culturally known by bird traders to have common local names that are based on distinctive behavior. For example, Acridotheres javanicus is known to have a local name 'jalak kebo', the local name has a relationship with the behavioral peculiarities of this bird species which is often seen by local community usually looking for foods, including insects with perched on the backs of buffalo (kebo or kebau). Some bird species of Family Nectrainiidae, such as Anthreptes sp, are known to have a local name as burung madu. Because bird species are often seen by local people in addition to looking for food in the form of flower insects, also likes for looking flower honey (madu bunga). Therefore, Anthreptes sp birds are known by residents as honey birds.

Meanwhile, Sitta frontalis is locally known as rambatan. The rambatan means creeping. Because the species of bird is often observed by local people predominant creeping on tree trunks or branches. Like Pasty bird traders, this bird species is culturally named by the Baduy community of South Banten as 'manuk saleser'. Saleser also means to creep, because the birds are often observed by the Baduy people has distinctive behavior usually creeping on the trunks and branches of trees in the forest areas of Baduy village (Iskandar et al. 2019 ${ }^{\mathrm{b}}$ ).

\section{Involving a bird song contest}

The bird species traded on the bird market can also be classified by bird traders (folk classification) into two main groups; the bird species normally involved in the bird song contest and bird species never involved in the bird song contest. Some bird species, including Zoothera citrina, Zoothera interpres, Copsychus malabaricus, Copsychus saularis, Serinus canarius and Agapornis sp. are known in involving in the birding contest in Indonesia. Meanwhile, other species are commonly used as birds keeping in each household and never involved in a bird song contest.

\section{Flying racing competition}

The bird species of dove-Columba livia can be culturally classified into 2 major groups, namely 'ordinary dove' (merpati biasa) and 'racing dove' (merpati balap). Ordinary dove groups are doves that are usually never involved in flying racing competition. Meanwhile, racing pigeons that are usually used for flying racing competitions. Moreover, racing doves may be divided into 2 categories, namely low flying racing dove and tall flying racing dove competition. The low flying racing doves are usually involved in low flying racing competition approximately 2-3 m above ground level. Meanwhile, tall flying racing competition doves are capable of flying 150 $\mathrm{m}$ above ground level (Zebua et al. 2016). Dove flying racing competition activities have many fans in the urban area. Therefore, doves are widely traded in bird markets (Figure 7).

The race begins, with two male bird doves off their cages at the departure point which is approximately $1 \mathrm{~km}$ from the landing site. The first male dove lands under the pit and touches the mat, to go to his partner to be the winner of the race. Then, continue the next round by doing the same thing as the previous work procedures. The winner of a flying racing competition usually takes several rounds. The winning bird competition has usually an increased selling price.

\section{Selling price}

Some bird traded in the bird market of Past which is based on selling price may be classified into 2 groups: bird species have low prices and bird species have high prices. The bird species of cheap birds are usually depending on the species and condition of birds. The non-song bird species of the Estrildidae family, including Lonchura leucogastroides, Lonchura punctulata, and Lonchura maja, have low prices. This is because these species of birds do not have beautiful feather colors, interesting singing sounds, and good attractive behavior. In addition, these cheap bird species are common species and the population is still abundant in rural ecosystems. 


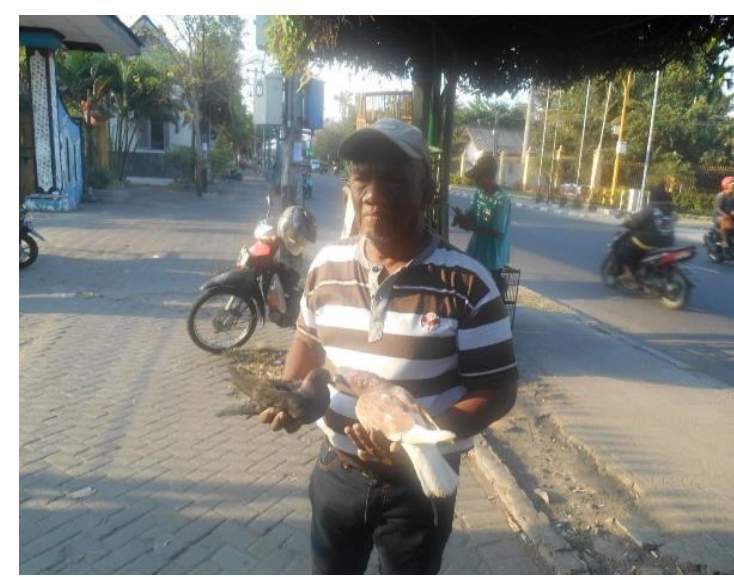

A

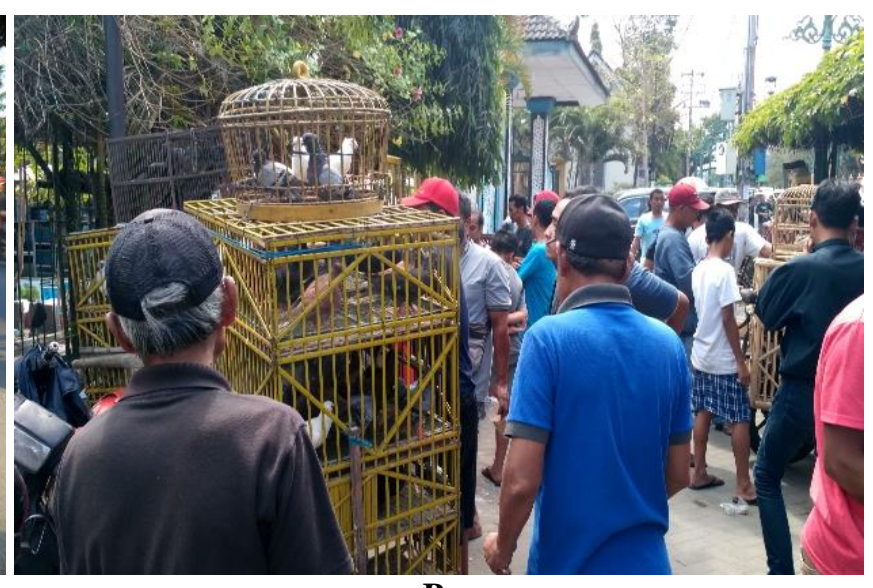

B

Figure 7. A dove trader is offering a pair of doves (Columba livia) (A) and bird buyers are watching the doves in their cage (B)

In addition to bird species, the cheap bird species are also determined by bird conditions. In general bird individuals have been freshly caught from nature and directly sold to bird markets as called burung bakalan have a low selling price. Therefore, although the bird species that are categorized as bird songs but they have been freshly caught usually have a low selling price. Because the individual birds must be cared for and adopted first by bird hunters or bird traders, and has high risk may die due to not able to adapt in bird keeping cages.

The selling price of a bird is also determined by gender. In general, male bird individuals, particularly for dimorphic birds, are more expensive than female individuals. Because the male birds have a more attractive feather color and have a harder various song and melodious. Therefore, some male bird species of songbirds are usually involved in the bird song contest.

The species of bird songs generally have a more expensive selling price compared to the species of nonsong birds. In addition, the species of birds that have beautiful feather colors, good singing, and attractive behavior also have expensive selling prices. Indeed, rare and protected bird species have high selling prices. Other factors, an individual tame bird that is usually called burung jadi, also have a high selling price compared to the same species of birds have just captured from nature (burung bakalan).

Some birds species, including Pycnonotus zeylanicus, Copsychus malabaricus, Copsychus saularis, Zoothera citrina, Zoothera interpres, and Chloropsis sonnerati which are commonly used for bird song contest have high selling prices on the bird market. Moreover, the species of birds that won in the songbirds contest were also more expensive in the bird market (Iskandar and Iskandar 2015). Wilderness

The bird species traded in the Pasty bird market which is based on wilderness may also be classified into two 2 categories: the wild birds that have been freshly captured from nature and still wild or semi-wild condition (burung bakalan) and bird species that have been temporarily adapted at home by bird hunters or bird traders that is usually called as a tame bird (burung jadi). Some bird captive breeding species, including Sternus canaria, Agapornis sp, and Melopasittacus undulatus are also categorized as tame birds. Besides, several birds species of birdsong contest, such as Copsychus malabaricus, Copschus saularis, and Zoothera citrina that have been successfully bred by bird breeders in the city, maybe categorized as a tame bird.

In general, the captive breeding species are usually given ring in their feet as indicated that birds are produced from bird captive breeding. Also, the birds are predominantly used for national the songbird contest, particularly birds that have been given a ring on their feet, indicating that the birds are produced from the captive breeding program. However, for the regional bird contest, especially at the local level, bird contestants are not required to use a ring on their feet. Therefore, the possibility of these birds is not the result of captivity breeding but the results of capture in nature.

As can be seen from the local bird names and bird classification that is undertaken by bird traders in Pasty bird market is similar to Sundanese ethnic of West Java in that it has local names and bird classification based on distinctive voice, morphological characteristic, special color, distinctive behavior, and habitat type (Iskandar et al. 2016; Iskandar 2017). The bird trader knowledge can be considered as TEK that is obtained by cultural transmission process by three methods; vertical transmission, horizontal and oblique transmission, with has characteristics, such as holistic, integrative, and situated within the broad cultural tradition (Ellen and Harris 2000; Hunn 2002; Pam 2017).

\section{Market chain system}

Since the bird market of Pasty was relocated from the traditional market of Ngasem, Yogyakarta in 2010, the dominant bird traders in Pasty are bird traders who moved from the Ngasem bird market. Many of them have been involving in bird trading for a long time. Many of the bird traders initially involved with their parents. At first, they 
had involved in trading activities since childhood, helping their parents to trade birds in the bird market. Then, when they are teenagers and adults continue their parents' business trading bird in the bird market. In addition, some bird traders change their livelihood. For example, before involving in bird trading, they have livelihoods other livelihoods, including Pedicab driver (tukang beca). But they decided to trade birds at the bird market because trading birds is considered to provide economic benefits.

Bird traders in the bird market of Pasty may be divided into two categories: the traders who have stalls and traders who do not have stalls. The traders who have stalls usually sell various commodities, including birds; bird feed; bird medicines; bird vitamins; and various items related to bird keepings, they sold them in their stall. Some items, including cages, can vary made of bamboo and wood, and iron cages. Nest can be a hiding place and a bird's nest, made of bamboo betung (Gigantochloa verticillata). Bird nest material is usually made of various materials, including pineapple leaf fibers which are considered good for canaries because of anti-lice; rice straw for chicken nests; coconut fiber for larwo bird nests, sengon sawn wood remnants for lovebird bird nests. Specifically for magpie nests made from pine branches collected from forest areas. Perched birds can be in the form of bonding rice stalks to perched bran.

While, various bird foods can be divided into two categories: natural food and non-natural food. Natural feeds of birds consist of banana of var. kepok, corn, brown rice, grain, millet, millet, caterpillars, hongkong caterpillars, crickets, kroto, and grasshoppers. Meanwhile, non-natural bird feed that is made by the bird feed industries. For example, the feed produced by SKM Kroto sari, the ingredients are made from milk, beans, honey, eggs; feed pur/top song; and bird medicines and vitamins, such as bird medicines in the form of anti-stress drugs for bird species, including Copsychus malabaricus, Copsychus saularis, Lanius schach, Ortomus ruficeps, Zosterops palpebrosus, Nectarinia spp, and Pyconotus goiavier. Unlike the bird traders who have stalls, the bird traders who do not have stalls, they usually trade birds in the green open space and on the edge of the main road in front of the pasty bird market complex.

Bird trading activities at the Pasty bird market are carried out every day, usually open from morning to evening. For bird sellers who have stalls, usually every morning at approximately 7.00 o'clock, they usually start to bring out bird cages to hang in front of their stalls in front of stalls that the birds can be dried in the morning sunshine. During the day, the cages of the birds are usually moved to hang in the shade of the stall building. Because the birds are hot by the sun. In the afternoon around 16.0017,00 o'clock bird cages are put in stalls and the stall are closed. So, the stall owners and their workers returned to their homes. The bird traders who do not have stalls that sell outside stalls, cage usually placed in open green spaces on the side of the street. Each morning they usually leave their homes carrying bird cages go-to bird market by motorbikes or bikes. Then in the afternoon, they return to their homes by bringing of birds that have not been sold.
The bird traders obtain the supply of various bird species for daily trading in the bird market from different ways, including obtaining bird supplies from local traders (pengepul) of the village, sub-district and district; supplies from bird breeders, and buying from other bird traders. Supply of birds from breeders, species of birds are limited, such as Serinus canaria, Agapornis sp, and Melopasittacus undulatus, as well as several local bird species that are usually competed in songbird contested that has been successfully bred by bird breeders or by bird farms.

Meanwhile, the main source of supply for local bird species traded in the Pasty bird market comes from illegal hunting from rural areas. There are 2 categories of supply of bird species from illegal hunting of rural areas. First, people from the city hunt for birds by going to the villages. They hunt birds in rural areas which are still found by many birds, such as in rural areas G. Merapi and G. Kidul of Yogyakarta. Second, bird hunters carried out by hunters from local rural areas who still have forest areas in the Yogyakarta region, and some rural areas outside of Yogyakarta, including the Dieng of Central Java province (Figure 8).

Both bird hunters of city and bird hunters of the local village usually hunt birds by using various techniques, including using nets, gum (pulut) that made from the sap of three of bendo (Artocarpus elaticus), jackfruit (Artocarpus heterophyllus), and rubber (Hevea brasiliensis). Catches of bird species are usually brought to their respective homes to be adapted in their homes for around 1-2 weeks. After the species of birds have been adapted for sufficient time, and the bird species have been rather tame, all the bird species are brought to the bird market to be sold to traders at the bird market.

The hunters of the local village people have involved in bird hunting may be as a part-time or full-time job, looking for birds in areas that still have many birds in their village. The village people who have involved in a part-time job, hunt birds particularly during the dry season when they do not busy in agricultural farming activities, while the village people who involved in a full-time job, usually hunt birds in the dry season as well as the wet season.

Based on ecological history, in the past local village, people used to hunt wild birds in their ecosystems were used for subsistence, including bird keeping in the cages of each household and local trading in villages. Nowadays, however, many local villagers have involved in hunting birds because almost every bird can be traded and can make money. Some of the bird hunters in the villages may also a double role that is besides being a bird hunter, also as a village middleman (pengepul) as bird collectors by buying birds from other bird hunters. All bird species that have been collected in the village, when the population number of catching birds is considered sufficient, all birds are brought to the bird market.

The bird traders from the rural, sub-district, and district area (pengepul) usually come to the bird market in the city once or twice a week on Sunday. In addition to bird sellers, some traders of natural bird feed, including pisang kapok (Musa x paradisiaca var kepok), caterpillars, crickets, and grasshoppers usually also come to the bird market in every 
Sunday. As a result, every Sunday the bird market in Pasty is usually crowded with bird traders and feed sellers from many regions to supply as well to sell both birds and bird feed. At the same many urban bird buyers also come to the bird market of Pasty would like to buy both birds and bird feed (Figure 9).

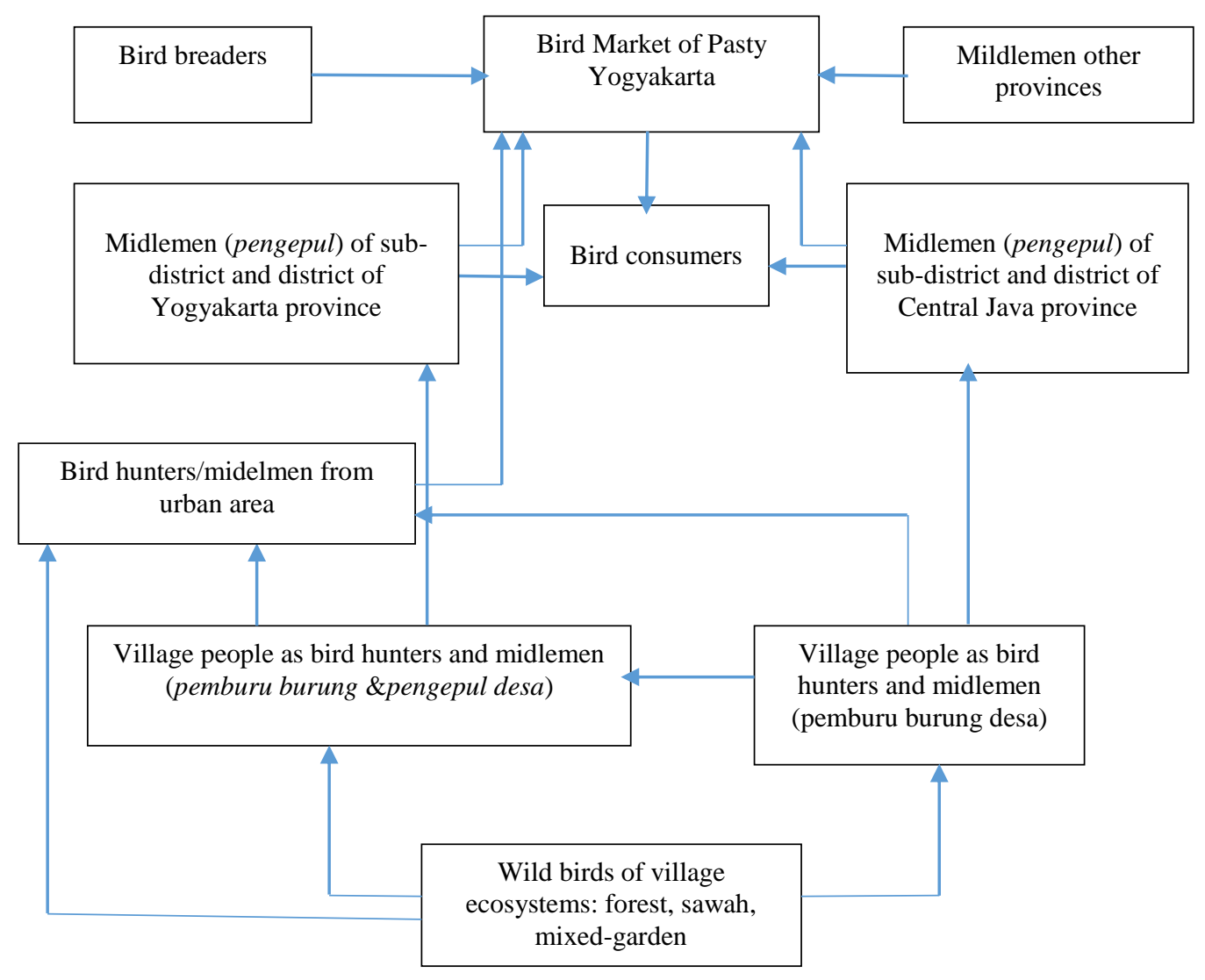

Figure 8. The market chain system of the bird market of Pasty, showing strongly interrelationship between the rural and urban area. Note: Village people as bird hunters and middlemen may be divided into two categories as full time and part-time in involving in bird hunting and bird-trading in the village; therefore, in the figure, the box is divided two

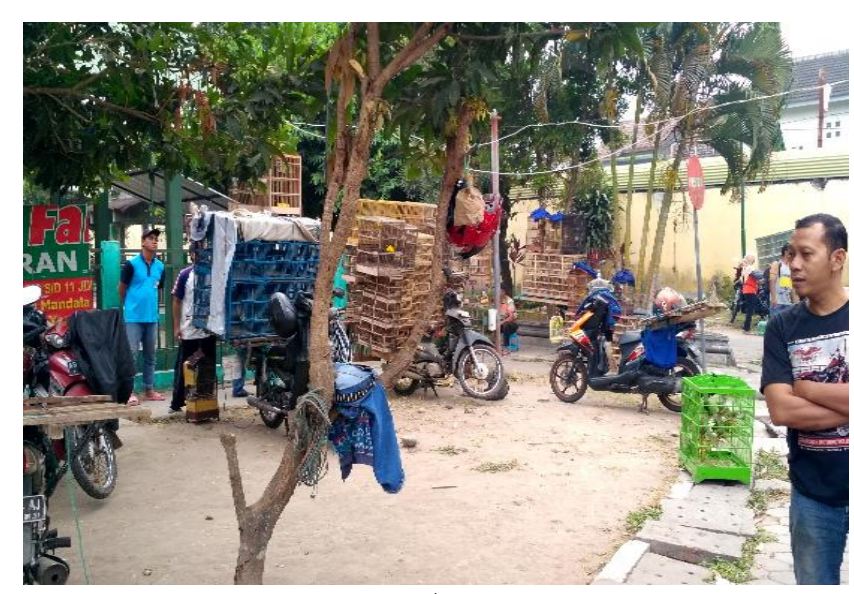

$\mathbf{A}$

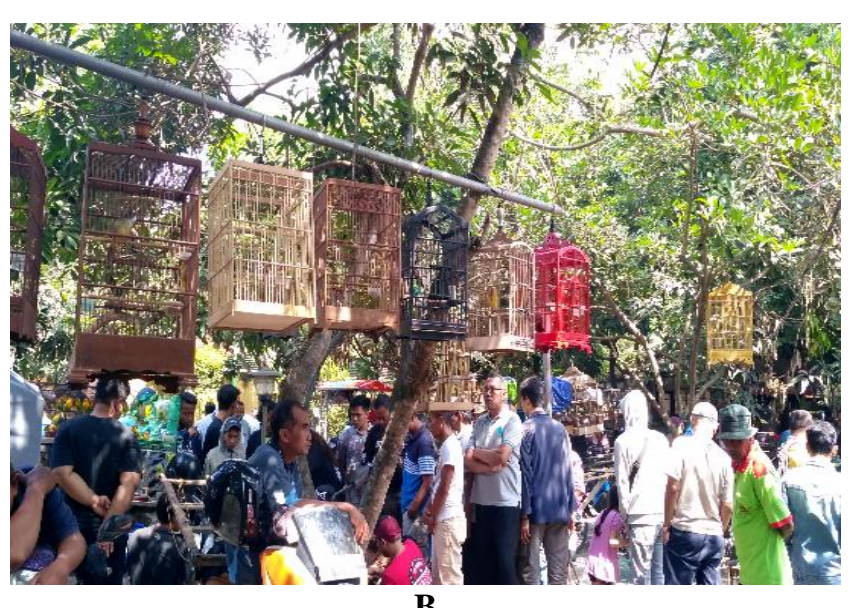

B

Figure 9. Each Sunday some bird traders from urban areas usually come to bird market for supplying birds that are caught in rural ecosystems (A), and the bird market is predominantly by bird consumers to buy birds (B) 
In addition to bird supply, various cage types are supplied by birdcage craftsmen from different regions of Central and East Java, including Klaten, Solo, Jepara, Wonosari, and Malang. Especially for iron bird cages are usually sent from Surabaya. Meanwhile, a place to look for branches and sprigs of pine as nest bird material, including taken from the Kali Urang forest area, Yogyakarta. The branches of pine are predominantly collected from the mountain area. This is because the pine branches collected from the coastal area are considered to be unfavorable due to they have high salt content which can cause the eggs of the birds to not hatch.

Based on bird trading activities in the bird market of Pasty, it can be revealed that trading of bird species in the bird market has provided economic benefits to many people, such as bird traders in the bird market, middlemen of bird collectors in villages and cities, village and city bird hunters, feed sellers in villages and cities, bird medicine and vitamin industries in cities, bird nest sellers in villages and cities, and bird breeders.

The trading bird species in the bird market, despite providing various economic benefits to many people, but the trading system of bird species that are not managed properly; including many protected bird species have been commonly sold in bird markets; undoubtedly may threaten the sustainability of many bird species in nature. Because the demand for rare and protected has increased, the price is higher. Consequently, many village people more interested to hunt the rare and protected bird to get income to fulfilling daily needs. The cause of hunting birds is more profitable than involving in agricultural labor, which was an increase in bird hunting undertaken by village people over time.

Therefore, if the hunting rate of rare or endangered species is higher than the natural rate of regeneration of these birds species, then, in the long run, these species will become extinct (Bennett and Robinson 2000). Indeed, based on the results of cross-studies culture in Indonesia and other countries shows that the factors of hunting and trade in bird species, in addition to habitat destruction or extinction, have been the main causes of the scarcity and even extinction of bird species in nature (Bennett and Robinson 2000; Bennett et al. 2002; van Balen et al. 2011; Fernandez-Ferreira et al. 2012; Chng et al. 2015; Harris et al. 2015; Irawati et al. 2016; Dai and Hu 2017; Shepherd and Cassey 2017; Setioko 2019; Hakim et al. 2020).

Implications of the results of this research appear several important things including that the bird trade in urban markets has a very complex relationship with the socio-economic and cultural system of rural populations. Rural people actually in the past utilized the species of birds strongly based on traditional ecological knowledge (TEK) and beliefs (Toledo 2002; Berkes 2008; Iskandar 2018). The beliefs of rural people, including beliefs in feared some birds, cause taboo to be caught and traded. Similarly, there are some forest areas in the village, which are considered as a sacred place (hutan keramat) by villagers, traditionally conserved by the rural people, which are important habitats for bird conservation. But, nowadays due to change in the belief systems, the intensive penetration of the market economy system, and the low economic level of the villagers. Consequently, there is no taboo system to protect bird species and protect sacred bird habitats. Many birds have been intensively hunted and traded both in villages and urban. Therefore, to protect the species of birds in Indonesia, it is necessary to pay attention to the socio-economic and cultural aspects of people in a rural area as well as urban areas. In general, the conservation of bird species which only emphasizes formal laws and regulations, empirical evidence shows that it is not effective in conserving bird species. Therefore, for bird conservation in nature, it is necessary to involve active participation from the community (Iskandar 2015).

Undoubtedly, ethnoornithology as transdisciplinary research and as sustainability science has been an important role in researching to develop scientific theories and practical results may be used for bird conservation based on active local people participation (Iskandar 2017; Pam 2017).

In conclusion, it can be said that the diversity of birds encountered in the bird market of Pasty is considered to be high, recorded 106 species representing 40 families. Although the high diversity considered to be high, in terms of the evenness of species is low. Only about only $27 \%$ of species classified as dominant and subdominant, while 73 $\%$ of species classified as non-dominant.

The common local names and folk bird classification are culturally based on bird vocalization, special colors, morphological characteristics, special habitats, involving bird song contests, flying racing competitions, selling prices, and wilderness. Most wild and semi-bird species are traded in the Pasty bird market are predominantly supplied from rural areas. Therefore, to maintain the sustainability of the bird trading system in many urban areas, the bird populations in rural ecosystems must be properly maintained. Indeed, the rural people as the main component in rural ecosystems, the active participant of rural communities must properly be involved in the conservation of birds.

\section{ACKNOWLEDGEMENTS}

The research on which both this research and this article is based would not have been possible without the financial support of the SIMLITABMAS, Ministry of Research and Technology for funding the research project of Dr. Budiawati Supangkat Iskandar MA, Decree Number 10/E1/KP.PTNBH/2019 and Agreement/Contract Number 5097c/UN6.G/LT/2019. We would also like to thank Dinas Perdagangan, Industri dan Pasar, Yogyakarta, Indonesia who permitted conducting this research. We would also like to thank informants of bird traders of the Pasty Bird Market, Yogyakarta who kindly provided valuable data for this research. 


\section{REFERENCES}

Albuquerque UP, de Lucena RFP, de Frietas Lins Neto EM. 2014 ${ }^{\mathrm{a}}$ Selection of research participants. In: Albuquerque UP, Cruz da Cunha LVF, de Lucena RFP, Alves RRN (eds). Methods, and Technique in Ethnobiology and Ethnoecology. Springer Science Business Media, New York.

Albuquerque UP, Monteiro JM, Ramos MA, de Amorim ELC, Alves RRN. 2014 ${ }^{\text {b }}$ Ethnobiological research in markets. In: Albuquerque UP, Cruz da Cunha LVF, de Lucena RFP, Alves RRN (eds) Methods, and Technique in Ethnobiology and Ethnoecology. Springer Science Business Media, New York.

Alves RRN. 2012. Relationships between fauna and people and the role of ethnozoology in animal conservation. Ethnobiol Conserv 1 (2): 1-35.

Alves RRN, Souto WMS. 2015. Ethnozoology: A brief introduction. Ethnobiol Conserv 4 (1): 1-13.

Adinugroho MM. 2013. Development Effort of Wildlife Market Dongkalan, Yogyakarta. [Report]. Faculty of Social Science, State University of Yogyakarta [Indonesian]

Ayat A, Tata HL. 2015. Diversity of birds across land use and habitat gradient in forests, rubber agroforest, and rubber Plantations of North Sumatra. Indon J forestry res 2 (2): 103-120.

Bashari H, Nurdin K. 2009. parrots are hunting and trading status in North Maluku 2008-2009. Bogor: Burung Indonesia. [Indonesian]

Bennett EL, Robinson JG. 2000. Hunting of Wildlife in Tropical Forests Implications for Biodiversity and Forest Peoples. The International Bank for Reconstruction and Development/The World Bank, Washington DC.

Bennett EL, Milner-Gulland EJ, Bakkar M, Eves HE, Robinson JG, Wilkie DS. 2002. Forum hunting the world's wildlife to extinction. Oryx 36 (4): 328-329.

Berkes F. 2008. Sacred ecology Second Edition. Routledge, London.

Carlson TJS, Maffi L. 2004. Introduction: Ethnobotany and conservation of biocultural diversity. In: Carlson TJS, Maffi L (eds). Ethnobotany and Conservation of Biocultural Diversity. The New York Botanical Garden Press, New York.

Chng SCL, Eaton JA, Krisnasamy K, Shepherd CR, Nijman V. 2015. In the Market for Extinction: An Inventory of Jakarta's Bird Markets Traffic Report Southeast Asia Regional Office, Selangor.

Cunningham AB. 2001. Applied Ethnobotany: People, Wild Plant Use, and Conservation. Earthscan, London.

Dai C, Hu W. 2017. Hunting strategies employed by bird hunters with an economic pursuit in the City of Guiyang, Southwest China. J Na Conserv 40: 33-41.

Department of Forestry. 2007. U Law Number 5 of 1990 Regarding the conservation of biological natural resources and their ecosystems. Department of Forestry, Jakarta. [Indonesian]

Ellen R, Harris H. 2000. Introduction. In: Ellen R, Parkes P, Bicker A (eds). Indigenous Environmental Knowledge and its Transformations: Critical Anthropological Perspectives. Hardwood Academic Publishers, Amsterdam.

Fernandez-Ferreira H, Medonca SV, Albano C, Ferreira FS, Alves RRN 2012. Hunting, use, and conservation of birds in North East Brazil. Biodivers Conserv 21: 221-244.

Hakim L, Abdoellah OS, Parikesit, Withaningsih S. 2020. Impact of agricultural type and hunting on bird communities of two villages in Bandung, West Java, Indonesia. Biodiversitas 21 (1): 57-66.

Harris JBC, Green JMH, Prawiradilaga DM, Giam X, Giyanto, Hikmatullah D, Putra CA, Wilcope DS. 2015. Using market data and expert opinion to identify overexploited species in the wild bird trade. Biol Conserv 187: 51-60.

Hoogerwerf A. 1949. The Avifauna of Bogor Botanical Garden Koninklijke Plantentuin van Indonesie, Bogor. [Dutch]

Hunn ES. 2011. Ethnozoology. In: Anderson EN, Pearsall D, Hunn, Turner N (eds). Ethnobiology. Wiley-Blackwell, New Jersey.

Irawati D, Arini D, Yuliantoro I. 2016. The typology and motivation of the owner community of Nuri Taulud as a protected bird in Karakelang island. Jurnal Penelitian Sosial dan Ekonomi Kehutanan 13 (1): 37-46. [Indonesian]

Iskandar J. 2014. Human and Environment with its Changes. Graha Ilmu, Yogyakarta. [Indonesian].

Iskandar J. 2015. The dilemma between hobbies and the bird trade business and bird conservation. In: Mardiastuti A, Mulyani YA (eds) Proceedings of the National Conference of Bird Researchers and
Observers in Indonesia. Faculty of Forestry, Bogor Agricultural Institute, Bogor, 13-14 February 2015. [Indonesian]

Iskandar J. 2017. Ornithology and Ethnoornithology. Plantaxia, Yogyakarta. [Indonesian]

Iskandar J. 2018. Ethnobiology, Ethnoecology, and Sustainable Development. Plantaxia, Yogyakarta. [Indonesian].

Iskandar $\mathrm{J}$ and Iskandar BS. 2015. The benefit of various birds in the song-bird contest and its impact on bird conservation in nature: A case study in Bandung, West Java. Pros Sem Nas Masy Biodiv Indon 1 (4): 747-752. [Indonesian].

Iskandar J, Iskandar BS, Partasasmita R. 2016. The local knowledge of the rural people on species, role, and hunting of birds: A case study in Karangwangi village, West Java, Indonesia. Biodiversitas 17 (2): 435 446

Iskandar BS, Iskandar J, Partasasmita R. 2019a . Hobby and business on trading birds: Case study in bird market of Sukahaji, Bandung, West Java, and Splendid, Malang, East Java (Indonesia). Biodiversitas 20 (5): 1316-1332.

Iskandar J, Iskandar BS, Nurjaman D. 2019 ${ }^{\mathrm{b}}$. Wildlife Diversity of Baduy South Banten for Ecotourism Interest. Plantaxia, Yogyakarta. [Indonesian].

Janiawati IA. 2012. Practice Report of Rare Fauna Conservation of Program III, Wildlife Trading. Faculty of Forestry, Gajah Mada University, Yogyakarta. [Indonesian]

Jepson P. 2010. Towards an Indonesian bird conservation ethos: Reflection form a study of bird-keeping in the cities of Java and Bali. In: Tideman S, Gosler A (eds). Ethnobiology: Birds, Indigenous People, and Society. Earthscan, London.

King BF, Dickinson EC. 1975. Field Guide Birds of South-East Asia. William Collins, London.

Mackinnon J, Phillips K, van Balen. 1992. Birds of Sumatra, Java, Bali, and Kalimantan. Puslibang LIPI, Bogor. [Indonesian].

Newing H, Eagle CM, Puri RK, Watson CW. 2011. Conducting Research in Conservation: Social Science Methods and Practice. Routledge, London.

Pam GA. 2017. Ethno-ornithology and Conservation: Traditional Ecological Knowledge TEK) of Birds Among the Mushere and the Conservation of the Dulu Forest in Mushere, Plateau State Nigeria. [Ph.D. Thesis]. University of Oxford, London.

Pangau-Adam M, Noske. 2011. Wildlife hunting and bird trade in Northern Papua (Irian Jaya), Indonesia. In: Tidemann S, Gosler A (eds). Ethno-ornithology: Birds, Indigenous People, Culture and Society. Earthscan, London.

Partasasmita R, Iskandar J. Malone N. 2015. Karangwangi people's (South Cianjur, West Java, Indonesia) local knowledge of species, forest utilization and wildlife conservation. Biodiversitas 17: 154-161.

Prakosa BH, Kurniawan N. 2015. Study on traded birds in bird market of Splendid, Malang City. J Biotropica 3 (1): 7-11.

Pratt TK, Beehler BM. 2015. Birds of New Guinea Second Edition. Princeton University Press, Princeton and Oxford, United Kingdom.

Setioko RAP. 2019. Study on the Kind and Conservation Status of Birds traded in Metro and Bandar Lampung Area. [Scription]. Biology Department, Faculty of Mathematics and Natural Sciences, University of Lampung, Lampung. [Indonesian].

Shepherd CR, Cassey P. 2017. Songbird trade crisis in Southeast Asia leads to the formation of IUCN SSC Asian songbird trade specialist group. J Indonesian Nat History 5 (1): 3-5

Suba RB, Rakhman A, Rustam. 2011. Captured trend of economically wild birds and its conservation implication: Case in Tanah Grogot, Paser District, East Kalimantan Province. Berita Biologi 10 (6): 797-806.

Sukmantoro W, Irham M, Novarino W, Hasudungan F, Kemp N, Muchtar M. 2007. List of Birds No.2. Indonesian Ornithologists's Union, Bogor. [Indonesian].

van Balen S, Eaton JA, Rheindt FE. 2011. Biology, taxonomy, and conservation status of the short-tailed green magpie Cissa [t.] thalassina from Java. Bird Conserv Int 23: 1-19.

Widodo W. 2007. Profile and perception of bird traders against the trade of the Perkeci Pelangi (Trichoglossus haematodus) and its conservation efforts. Berkala Penelitian Hayati 13: 67-72. [Indonesian].

Wiedarti S, Moerfiah, Sudrajat, Iqbal GM. 2016. The diversity of bird species that are traded in the bird market in the city of Bogor, Indonesia. AIP Conference Proceedings. DOI: 10.1063/1.4953530.

Zebua FE, Riyanti, Kurtini T. 2016. Different characteristics of male bode and Columba livia local male. Jurnal Ilmiah Peternakan Terpadu 4 (3): 244-248. [Indonesian] 
BIODIVERSITAS

Volume 21, Number 6, June 2020

Pages: 2586-2602
ISSN: 1412-033X

E-ISSN: 2085-4722

DOI: 10.13057/biodiv/d210631 